\title{
Liebhard Egkenfelder und die Musikhandschriften in Pressburg in der zweiten Hälfte des 15. Jahrhunderts
}

\section{Liebhard Egkenfelder and the music manuscripts in Bratislava in the second half of the $15^{\text {th }}$ century}

Eva Veselovská / eva.veselovska@savba.sk

Institute of Musicology, Slovak academy of sciences, Bratislava, SK

\begin{abstract}
Almost all preserved musical manuscripts from the 15th century from Bratislava belonged to the Library of Bratislava Chapter. A small part of manuscripts and fragments came from other parish, monastery and school libraries or from private collections. Complete liturgical codices and fragments are a testimony of the multicultural background of customers, makers, and users. The most precious materials from the second half of the 15th century are the liturgical manuscripts of the Bratislava Chapter - Bratislava Antiphonary Ila and IIb (Strigonium liturgy, Austrian scriptorium and illumination tradition) and Bratislava Antiphonary III (Strigonium liturgy, Buda-Renaissance scriptorium in combination with the Austrian illumination school). One of the most interesting personalities from Late Middle Ages Bratislava was a notary Liebhard Egkenfelder. He was the main scriptor in town between 1441 and 1456. He was an extraordinarily erudite layman and a great humanist representative. He owned a large private library with codices from various scientific disciplines. He himself copied several manuscripts. Amongst the codices he wrote "with his own hand" we can find material regarding both liturgical and secular music culture.
\end{abstract}

\section{Key words}

middle ages, musical manuscript, Bratislava, Liebhard Egkenfelder 
Der folgende Beitrag möchte eine Vorstellung von der spätmittelalterlichen Schriftkultur in Bratislava (dt. Pressburg, ung. Pozsony) durch die bestimmte Musikquellen und eine Persönlichkeit (Liebhard Egkenfelder) vermitteln. Fast alle erhaltenen Musikhandschriften des 15. Jahrhunderts aus Pressburg gehörten der Pressburger Kapitelbibliothek. Eine sehr kleine Anzahl kompletter Handschriften oder Fragmente stammen aus anderen Pressburger Pfarr-, Kloster- oder Schulbibliotheken. ${ }^{1}$ Geringe Nummer der Kodizes war Bestandteil privater Sammlungen (z. B. L. Egkenfelder).

Vom Aspekt der politischen Geschichte her deckt sich die Periode der zweiten Hälfte des 15. Jahrhunderts mit den Regierungsjahren des ungarischen Königs Mathias Corvinus (die Regierungszeit: 1458 -1490). In der Regierungszeit von Matthias begann die erste spürbare Durchdringung der Renaissance-Impulse aus Italien in einige ungarländische Kulturzentren. Vor allem zwei Kanäle sind zu benennen: der königliche Hof (dank der Heirat mit Beatrix von Aragonien) und die Höfe einiger Prälaten (vor allem in Gran). ${ }^{2}$

\section{Spätmittelalterliche Schriftkultur im Pressburger Kollegiatkapitel in der zweiten Hälfte des 15. Jahrhunderts}

Für das Kollegiatkapitel in Pressburg bedeutete diese Periode den Höhepunkt in ihrer mittelalterlichen Geschichte. Die Institution sorgte sich auch für den Bestand der Universität - Academia Istropolitana (1465 - 1488). ${ }^{3}$ Die Kapitel konnte (wenigstens am Anfang) bei der verantwortungsvollen Sorge um die Universität mit königlicher und erzbischöflicher Unterstützung rechnen. Es ist natürlich, dass sich die wirtschaftliche und intellektuelle Blüte auch auf die Position der Pröpste und des Kapitels auswirkte. Während die Kanoniker auch aus bürgerlichen Familien stammten, waren die Pröpste im späten Mittelalter meistens adeliger Herkunft. Beide Pröpste aus der Zeit Mathias Corvinus hatten eine Karriere auf dem Königshof hinter sich. Georg Schönberg (1455 - 1486) war ursprünglich Notar, später war er der Propst auch ein königlicher Botschafter und Diplomat. ${ }^{4}$ Anton von Sánkfalva (1486 - 1498) war seit 1468 ein Würdenträger in der königlichen Kanzlei. ${ }^{5}$ Die Pressburger Kapitelbibliothek und ihre kirchliche Büchersammlung wurden im Laufe des Mittelalters durch die Tätigkeit der Pröpste,

1 SOPKO, Július. Stredoveké latinské kódexy v slovenských knižniciach. Martin: Matica slovenská, 1981; SOPKO, Július. Stredoveké latinské kódexy slovenskej proveniencie v Mad’arsku a Rumunsku. Martin: Matica slovenská, 1982.

2 ŠEDIVÝ, Juraj. Mittelalterliche Schriftkultur im Pressburger Kollegiatkapitel. Bratislava: Chronos, 2007, S. 178-201.

3 ŠEDIVÝ, Juraj. Beiträge zur mittelalterlichen deutschprachigen Schriftkultur in der Slowakei I. Bratislava: Univerzita Komenského, 2019.

4 HLAVAČKOVÁ, Miriam. Juraj zo Schönbergu. Bratislavský prepošt v službách cisára a krála. Bratislava: VEDA - Historický ústav SAV, 2015.

5 HLAVAČKOVÁ, Miriam. Kapitula pri Dóme sv. Martina. Intelektuálne centrum Bratislavy v 15. storoči. Bratislava: Pro Historia, 2008; HLAVAČKOVÁ, Miriam. Vzdelanostná úroveň členov Bratislavskej kapituly v 15. storočí. Historický časopis 2001, 49, Nr. 3, S. 433-451. 
der Kanoniker bzw. des reichen Pressburger Bürgertums geschaffen. Für die liturgische Praxis in der St. Martins-Kirche wurden Mess- und Offizienkodizes in den großen Skriptorenwerkstätten im mittelalterlichen Ungarn im Ausland in Auftrag gegeben bzw. von heimischen oder reisenden Skriptoren, Illuminatoren und Notatoren in Pressburg selbst hergestellt. ${ }^{6}$ Viele Handschriften gelangten in die Kapitelbibliothek als Schenkungen oder durch testamentarische Nachlässe. ${ }^{7}$

Die soziale Herkunft der Stifter zeigt die typischen Veränderungen des Spätmittelalters: im 14. und am Anfang des 15. Jahrhunderts stammten die Auftraggeber, bzw. Stifter der Handschriften aus klerikalen Kreisen, nun sind es Bürger und ihre Frauen.

Belegt sind die Donationen z. B.: Liebhard Egkenfelders (1455/1457), der Familie Liszt (1478 - die Witwe des Pressburger Richters und Bürgermeisters Nicolaus List), eines Johann Pottenberger (1480) sowie der Witwe des Richters Wenzel Rosentaler Magdalena Rosentaler (1488), Margaret Schickenin stiftete im Jahre 1489 ein Messbuch, Barbara Aignerin schenkte 1491 dem Franziskanerkloster in Holíč ein Messbuch. 1489 schenkte an das Kapitel ein Missal für die Stiftung beim Wolfgang-Altar auch Pressburger Gespan. ${ }^{8}$

Die Gesellschaftsschicht der vermögenden Pressburger Familien und die Pressburger Chorherren entwickelten verschiedene wirtschaftliche und kulturelle Kontakte miteinander.

Das Leben der herausragenden Bürger der Stadt und der geistlichen Vertreter des Kapitels hatte eine direkte Einwirkung auf die Entstehung, Bestellung und Ausstattung von handschriftlichen Büchern. Im Laufe des 14. und am Beginn des 15. Jahrhunderts stand die Produktion der Pressburger Handschriften im Zeichen einer gewissen regionalen Eigenständigkeit. In den einzelnen Komponenten der Handschriften (künstlerische Ausschmückung, Notation) wurden abwechselnd böhmische bzw. österreichisch-mährische Elemente verwendet. ${ }^{9}$

Die Buchkultur in Pressburg erlebte erste Blüte in der ersten Hälfte des 15. Jahrhunderts, als König Sigismund (Regierungszeit: 1387-1437) die Stadt zu seiner Residenz machte. Neben der Inspiration durch die böhmische Buchmalerei ${ }^{10}$ oder durch

6 VESELOVSKÁ, Eva. Mittelalterliche liturgische Kodizes mit Notation in den Archivbeständen von Bratislava. Ed. Musaeum Musicum. Bratislava: Slovenské národné muzeum - Hudobné muzeum, 2002; VESELOVSKÁ, Eva - ADAMKO, Rastislav - BEDNÁRIKOVÁ, Janka. Stredoveké pramene cirkevnej hudby na Slovensku. Bratislava: Slovenská muzikologická spoločnost’ - Ústav hudobnej vedy SAV, 2017.

7 MAJOROSSY, Judit - SZENDE, Katalin (eds.). Das Pressburger Protocollum Testamentorum 1410 (1427)-1529. Teil 1: 1410-1487. Wien: Böhlau Verlag, 2010.

8 HLAVAČKOVÁ, Miriam. Oltárne benefíciá v bratislavskom Dóme sv. Martina v 15. storočí. In Galéria, 2001, Bratislava: Slovenská národná galéria, 2001, S. 85-99.

9 BURAN, Dušan. „Böhmisch - slowakisch - österreichisch“? Die Malerei in Pressburg (Bratislava). In Böhmische Buchmalerei des 14. und 15. Jahrhunderts. Purkersdorf: Verlag Brüder Hollinek, 2012, S. 45-55; VESELOVSKÁ, Eva. Der böhmische Einfluß auf mitteleuropäische Musikhandschriften des 14. und 15. Jahrhunderts unter Berücksichtigung illuminierter Codices. In Böhmische Buchmalerei des 14. und 15. Jahrhunderts. Purkersdorf: Verlag Brüder Hollinek, 2012, s. 95-115.

10 THEISEN, Maria - JENNI, Ulricke (eds.). Mitteleuropäische Schulen IV (ca. 1380-1400). Hofwerkstätten König Wenzels IV. und deren Umkreis. Wien: Verlag der Österreichischen Akademie der Wissenschaften, 2014. 
die böhmische Notation ${ }^{11}$ nach 1400, welche gleichzeitig allen benachbarten Regionen Böhmens eigen ist, gewinnt später in den Pressburger Handschriften ein anderer Stil die Oberhand. ${ }^{12}$ Im Laufe des 15 . Jahrhunderts verstärkten sich die Kontakte Pressburgs zu Wien und Niederösterreich. Dies führte zu der Tätigkeit mehrerer bedeutender österreichischer Illuminatoren und ihrer Werkstätten in Pressburg (Meister Michael, ${ }^{13}$ Ulrich Schreier). ${ }^{14}$ Weitaus weniger kommt unter den Pressburger Handschriften der zweiten Hälfte des 15. Jahrhunderts der Einfluss der Kunst des Ofner Hofes von Matthias Corvinus zum Vorschein. Diese Erkenntnis ist äußerst überraschend, da König Matthias 1465 immerhin die Universität Academia Istropolitana in Pressburg gegründet hat.

Im Kulturleben der Stadt dominierte in der zweiten Hälfte des 15. Jahrhunderts die Tätigkeit des Pressburger Propstes Georg von Schönberg. Propst Schönberg und der Stadtpfarrer und Kanoniker Johannes Han de Wep gaben in den siebziger und achtziger Jahren des 15. Jahrhunderts mehrere liturgische Handschriften in Auftrag, was in einer reichen Skriptorentätigkeit resultierte. Unter dem Einfluss der österreichischen Buchmalerei (Werkstatt von Ulrich Schreier) entstanden in der zweiten Hälfte des 15. Jahrhunderts die Pressburger Antiphonarien IIa (EC Lad. 4, Staatsarchiv Pressburg) und IIb (SNA 4, Slowakisches Nationalarchiv), zwei repräsentative Musikhandschriften (Graner liturgische Tradition), die von lokalen Einflüssen der österreichischen Notationstradition geprägt sind. Hinsichtlich der Notation knüpft die Metzer-gotische Schrift des Pressburger Antiphonars IIa und IIb an die feinere Form der Pressburger Antiphonarien $I$ (EC Lad. 3 Staatsarchiv Pressburg) und IV (SNA 2 Slowakisches Nationalarchiv) und vor allem an die zweite Notation des 4-bändigen Klosterneuburger Antiphonars Cod. $65-68$ an. $^{15}$

Die Metzer-gotische Notation des zweiten Notators des Pressburger Antiphonars IIb nähert sich den Notationselementen auch einer anderen Klosterneuburger Handschrift, dem Officium hl. Leopold Austria laetare Cod. 59 (datiert nach 1490). ${ }^{16}$

11 VESELOVSKÁ, Eva. Bohemian Notation in Slovakia in the Middle Ages. Hudební věda, 2012, Jahrg. 49, Nr. 4, S. 337-376.

12 BURAN, Dušan. Fragmente illuminierter gotischer Handschriften aus zwei slowakischen Archiven. In Biblos. Beiträge zu Buch, Bibliothek und Schrift Europa, Die Nationalbibliotheken der neuen EU-Länder. Wien: Phoibos Verlag, 2004, S. 67-79.

13 RISCHPLER, Susanne. Der Illuminator Michael. Codices Manuscripti. Supplementum 1, 2009. Purkersdorf: Verlag Brüder Hollinek, 2009. Zwei Wappenbriefe König Sigismunds für Pressburg aus dem Jahr 1436, Sign. 1006, 1007, Archiv der Stadt Pressburg. Pressburger Antiphonar IV, Sign. 2, Slowakisches Nationalarchiv.

14 Pressburger Antiphonar IIa (EC Lad. 4, Staatsarchiv Pressburg) und IIb (SNA 4, Slowakisches Nationalarchiv), Pressburger Antiphonar III (EC Lad. 6, Staatsarchiv Pressburg). Zur selben Zeit wurde auch das Pressburger Missale aus dem Jahr 1377 nachträglich verziert (Biblioteca Națională a României-filiala Batthyaneum, Cod. R. II. 134). Der Kanonbrief (f. 119) wurde im Auftrag des Kanonikers Johannes Han direkt in der Wiener Werkstatt des Ulrich Schreier illuminiert.

15 HAIDINGER, Alois. Bemerkungen zur Entstehung des Großen Antiphonars Klosterneuburg, Stiftsbibliothek, Cod. 65-68. In Böhmische Buchmalerei des 14. und 15. Jahrhunderts. Purkersdorf: Verlag Brüder Hollinek, 2012, S. 73-80; HAIDINGER, Alois. Katalog der Handschriften des Augustiner Chorherrenstiftes Klosterneuburg. Teil 1: Cod. 1-100. Wien: Österreichische Akademie der Wissenschaften, 1983.

16 VESELOVSKÁ, Eva. Métsko-gotická notácia na Slovensku v období stredoveku. Musicologica Slovaca, 2018, Jahrg. 9 (35), Nr. 2, S. 165-199. 


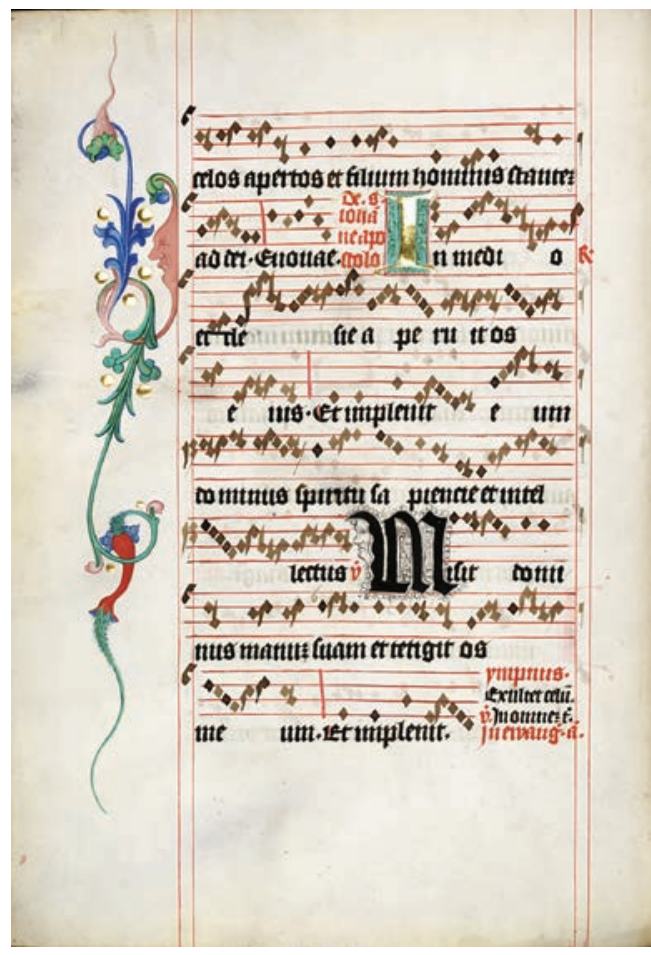

Abb. 1 Pressburger Antiphonar IIb SNA 4 (Slowakisches Nationalarchiv,

$$
\text { f. } 2 v) .{ }^{16}
$$

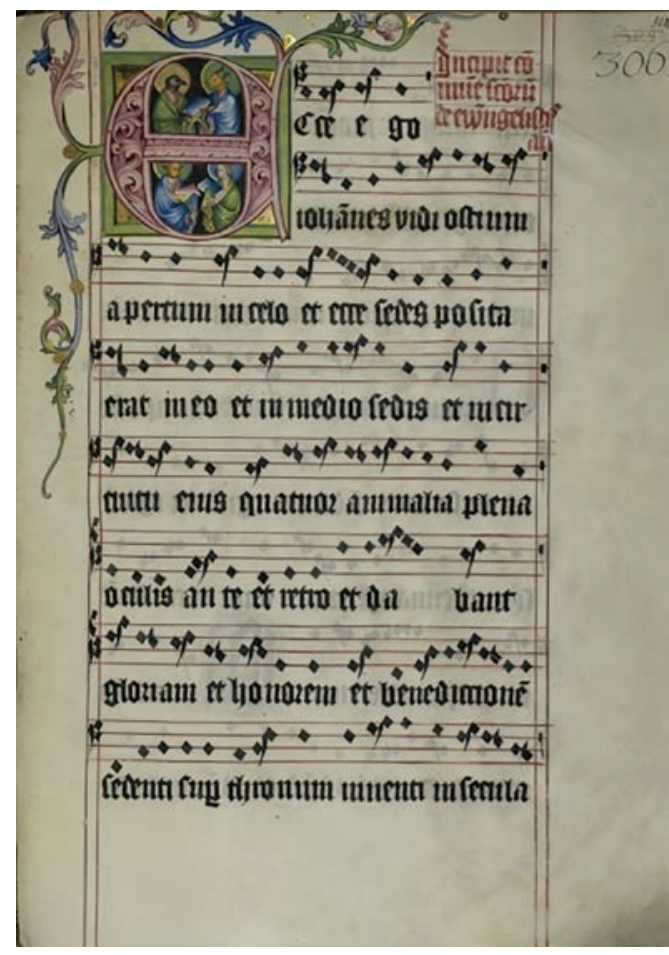

Abb. 2 Klosterneuburger Antiphonar Cod. 65 (Augustiner-Chorrherrenstift Klosterneuburger Antiphonar Cod. 65 (Augustiner-Chorrherrenstift Klosterneuburg, Stiftsbibliothek Klosterneuburg $\mathrm{CCl} 65$, f. 302r). ${ }^{17}$

Die veränderte Ästhetik der Kodizes vom Ende des Mittelalters nach dem Vorbild der italienischen Renaissance und des königlichen Skriptoriums in Buda wird einzig und allein nur durch das Budaer/Pressburger Antiphonar III (EC Lad. 6, Staatsarchiv Pressburg) dokumentiert. Ausschmückung, Schrift und Notationsstrukturen wurden durch die humanistischen Tendenzen und Usancen im Ofner Skriptorium beeinflusst. ${ }^{19}$ In dem Kodex taucht eine spezifische Notation auf, die als Folge der Reformprozesse in der Buchkultur der zweiten Hälfte des 15. Jahrhunderts zu betrachten ist. Diese Kontaktnotationsschrift, die nicht als reine Metzer-gotische Notation angesehen werden kann, enthält mehrere archaische Strukturelemente der Graner Notation. Janka Szendrei

\footnotetext{
17 http://cantus.sk/image/6157

18 http://manuscripta.at/diglit/AT5000-65/0606/image?sid=cd29825413de5f69972d858b56cd8cd5

19 BURAN, Dušan. Neznámy budínsky (?) iluminátor a maliar z okruhu Ulricha Schreiera. In RUSINA, Ivan et al. (eds.). Renesancia: umenie medzi neskorou gotikou a barokom: Dejiny slovenského výtvarného umenia. Bratislava: Slovenská národná galéria - Slovart, 2009, S. 873-874.
} 
bezeichnete diesen Notationstyp als Metzer-gotische Graner Mischnotation. ${ }^{20}$ Dieses Mischzeichensystem war ein vielbenutzter Notationstyp in der zweiten Hälfte des 15. und am Beginn des 16. Jahrhunderts. Es wurde vor allem in den zentralen ungarischen Skriptorenwerkstätten verwendet Gran und Buda verwendet. ${ }^{21}$

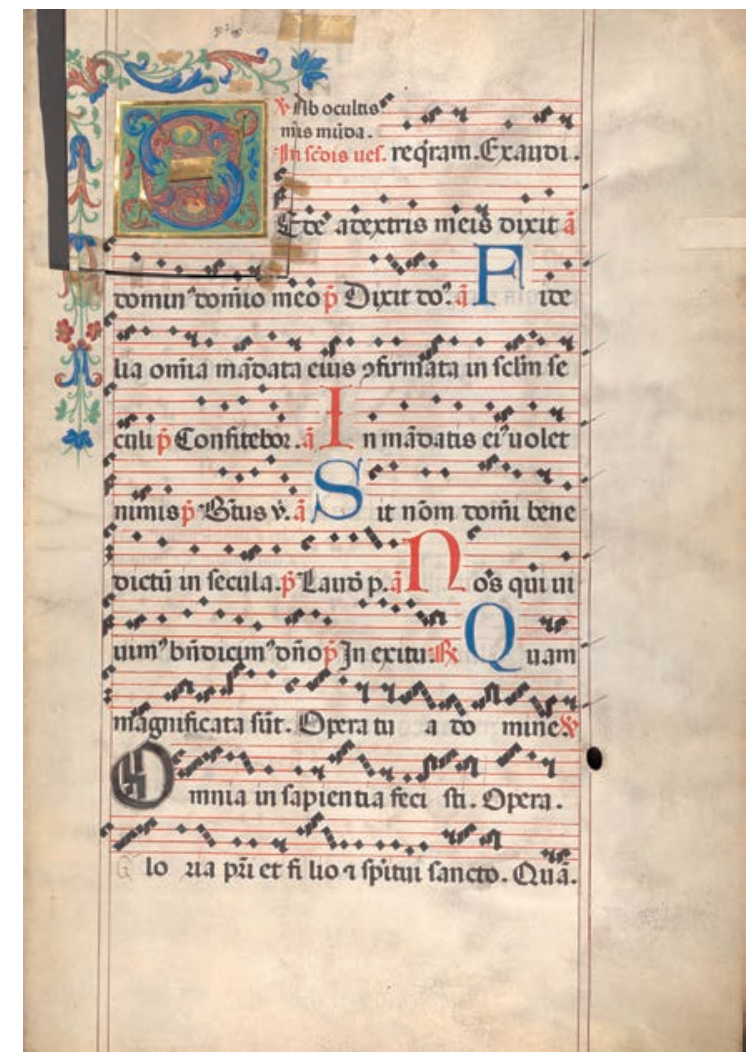

Abb. 3 Pressburger Antiphonar III EC Lad. 6

(Staatsarchiv Pressburg, f. 30r).

20 SZENDREI, Janka. Die Geschichte der Graner Notation. Studia Musicologica Academiae Scientiarum Hungaricae 1988, 30, S. 5-234.

21 SZENDREI, Janka. A magyar középkor hangjegyes forrásai. Budapest: MTA Zenetudományi Intézet, 1981, C 8: Antiphonale Strigoniense I. Esztergom, Főszékesegyházi Könyvtár [Bibliothek der Kathedrale Esztergom], Mss. I. 3, C 9: Antiphonale Strigoniense II. ebd. Mss. I. 3; C 45: Graduale Strigoniense Francisci de Futhak, Istanbul, Topkap Seray 2429. 


\section{Fragmente des Ofner/Pressburger Antiphonars III in der Österreichischen Nationalblibliothek Wien}

In der Österreichischen Nationalbibliothek Wien (weiter ÖNB) wurde seit 2008 eine komplexe Untersuchung der mittelalterlichen Musikmaterialien durchgeführt. Während der zwei dreijährigen Projekte wurden unter der Leitung von Dr. Alexander Rausch 367 Signaturen bearbeitet, von denen heute im Rahmen des Buchkatalogs 108 vollständige oder teilweise erhaltene Kodizes bearbeitet sind. Ausgewertet wurden ebenso über 670 Fragmente. ${ }^{22}$ Die Handschriften stammen aus dem Zeitraum vom Ende des 9. bis zum Beginn des 16. Jahrhundert und sind von unterschiedlicher Provenienz (Österreich, Böhmen, Polen, Frankreich, Deutschland, Italien, heutige Slowakei und Ungarn usw.). Erschienen sind mehrere wissenschaftliche Publikationen, Studien und erschlossen wurde eine Internetdatenbank der bearbeiteten Handschriften, der die bearbeiteten Materialien laufend geliefert wurden. ${ }^{23}$ Im Rahmen mehrerer Fonds der ÖNB befindet sich auch eine Gruppe von Fragmenten aus Ungarn, die Robert Klugseder als Gruppe GNA 3 bezeichnete. In dieser Gruppe von Fragmenten sind derzeit 32 Fragmente eingeordnet, die in Zusammenarbeit mit der ungarischen Musikhistorikerin Janka Szendrei ausgewertet wurden. ${ }^{24}$ Außer den erwähnten 32 Signaturen der Gruppe GNA 3 wurden dank der Empfehlung von Dr. Fridrich Simader weitere 7 Signaturen des Sanktorales dieser Handschrift aus den Einbänden der Kodizes Cod. 2917, Cod. 2918 und aus 5 hebräischen Handschriften Cod. Hebr. 5, Cod. Hebr. 37, Cod. Hebr. 67, Cod. Hebr. 70, Cod. Hebr. 71 bearbeitet. Da sich in mehreren der analysierten Signaturen inhaltliche Anmerkungen und Verweise der Person Sebastian Tengnagels befanden, kann folgende Hypothese ausgesprochen werden. Es ist höchstwahrscheinlich, dass auf Weisung des Wiener Präfekten der Hofbibliothek in Wien Sebastian Tengnagel Fragmente des Ofner/Pressburger Antiphonars III auf verschiedenen Handschriften und Drucken verwendet wurden, die sich heute in der Nationalbibliothek in Wien unter verschiedenen Signaturen und in mehreren Fonds befinden. Tengnagel (1573-1636) war Präfekt der Hofbibliothek in den ersten Jahrzehnten des 17. Jahrhunderts (1608-1633). In den Jahren 1609/1610

22 KLUGSEDER, Robert. Medieval musical manuscripts and fragments in the Austrian National Library (ÖNB), Vienna. In Cantus Planus. Papers read at the 16th Meeting Vienna, 2011. Wien: Verlag Brüder Hollinek, 2012, S. 192-201.

23 KLUGSEDER, Robert - RAUSCH, Alexander - ČIZMIĆ, Ana - CHARVAT, Vera Maria -VERHAAR, Oscar -VESELOVSKÁ, Eva - ZÜHLKE, Hanna. Katalog der mittelalterlichen Musikhandschriften der Österreichischen Nationalbibliothek Wien. In Codices Manuscripti E Impressi. Purkersdorf: Verlag Brüder Hollinek, 2014, Supplementum 10. http://e-book.fwf.ac.at/o:599.

24 Die Datenbank cantusplanus.at veröffentlicht z. Zt. 32 Signaturen (GNA 3), die den gleichen Skriptorenund Notatorenursprung dokumentieren wie das Ofner/Pressburger Antiphonar. Alle Bruchstücke stammen aus demselben Zeitraum (Ende des 15. Jahrhunderts, 1480-1495) und mit größter Wahrscheinlichkeit auch aus einer Handschrift (sicherlich aus einer Skriptorenwerkstatt). Sie weisen die gleichen kodikologischen und paläographischen Parameter auf. Diskutabel ist nur die Signatur Cod. 11.777, die entweder nicht in dieses Antiphonar gehörte, oder den Schluss der Handschrift bilden konnte. Zwei Fragmente dieser Signatur dokumentieren als einzige 12 Notenzeilen, wobei die Maße des Notationssystems kleiner ist als im Falle des Ofner/ Pressburger Antiphonars III. Die Höhe einer Notenzeile ist ca. 16-17 mm, der Zwischenraum misst ca. $5 \mathrm{~mm}$. 
verfasste er ein Verzeichnis der Bücher der Hofbibliothek in Wien. Mehrere Signaturen aus dieser Bibliothek, die in Fragmente des Ofner/Pressburger Antiphonars III (Cod. Hebr. 5, 37, 67, 70, 71 und die Fragmente 863, 866, 874, 875 und 880) gehüllt sind, enthalten inhaltliche Anmerkungen von Sebastian Tengnagel. Es ist daher sehr wahrscheinlich, dass diese Bände in Wien gerade während Tengnagels Wirken geschaffen wurden. Wir nehmen an, dass das Pressburger Kapitel den zweiten Teil der Handschrift um das Jahr 1600 (spätestens in den ersten Jahrzehnten des 17. Jahrhunderts) einer Wiener Buchbinderwerkstatt zur Verfügung stellte, die Einbände für jüngere Bücher, Handschriften oder Amtsbücher verschiedener Institutionen oder Einzelpersonen anfertigte. Auf den Fragmenten aus Wien sind verschiedene Teile des Sanktoralteils erhalten geblieben (Feste der Heiligen: Fabian und Sebastian, Agnes, Pauli Bekehrung, Agatha, Dorothea, die Verkündigung der Jungfrau Maria, Johannes der Täufer, Ladislaus von Ungarn, Johann ante Portam Latinam, Johann und Paulus, Anna, Maria de Nive, Verklärung des Herrn, Laurentius, Mariä Himmelfahrt, Stephan von Ungarn, Allerheiligen, Translatio Adalberti, Elisabeth von Ungarn, Katharina, Andreas, Nikolaus, Adalbert, Otmar) und Commune sanctorum (Feste der Apostel, eines Märtyrers, eines Bekenners, der Jungfrauen; Invitatorien zu verschiedenen Teilen des liturgischen Jahres). Die erhaltenen Quellen dokumentieren in mehreren Fällen spezifische und einzigartige Gesänge (Offizium der hl. Dorothea, des hl. Königs Ladislaus, der hl. Anna, Maria de Nive, des hl. König Stephan, Translatio Adalberti, der hl. Elisabeth und der hl. Katharina).

Das Ofner/ Pressburger Antiphonar III war Bestandteil der reichen Bibliothek des Pressburger Kapitels, obwohl es vermutlich nicht zu ihrem „Grundrepertoire“ gehörte. ${ }^{25}$ Es ist in einem außerordentlich guten Zustand erhalten, was von seiner geringen Benutzung zeugt. Die Geschichte dieser Handschrift ist durch das häufige Herausschneiden von Blättern geprägt. ${ }^{26}$

Die Fragmente der ÖNB, aber auch des St. Adalbert-Vereins, des Archivs des Slowakischen Nationalmuseums SNM und der Ungarischen Nationalbibliothek in Budapest ${ }^{27}$ sind trotzdem diesem Fakt sehr wichtig, weil sie die einzigartige Graner liturgische Tradition dokumentieren.

25 Im Gegensatz zu dem besprochenen Antiphonar zeugen die Pressburger Antiphonarien $I$ und $I V$ von einem außerordentlich häufigen Gebrauch mit zahlreichen Anmerkungen, beschädigten und abgeriebenen Ecken der einzelnen Folien.

26 Die Fragmente des Ofner/ Pressburger Antiphonars III befindet sich heute auch in der Ungarischen Nationalbibliothek in Budapest, im Verein des hl. Adalbertus in Trnava und im Archiv des Slowakischen Nationalmuseums. VESELOVSKÁ, Eva. Catalogus fragmentorum cum notis musicis medii aevi e civitate Tyrnaviensi. Catalogus fragmentorum cum notis musicis medii aevi in Slovacia. Tomus IV. Bratislava: Ústav hudobnej vedy SAV, 2015, S. 56-60; VESELOVSKÁ, Eva. Fragmente des Budaer Antiphonars im St. Adalbert-Verein Trnava und im Archiv des Slowakischen Nationalmuseums. Studia Musicologica, 2015, Jahrg. 56, Nr. 2-3, S. 233-246.

27 DOBSZAY, László. A »Budai antiphonále« megtalált töredékei. Zenetudományi dolgozatok. Budapest: MTA Zenetudományi Intézet, 1978, S. 35-39; MIKÓ, Árpád. Fáncsi Imre armálisa (1511) és két Budán illuminált, kottás díszkódex. Ars hungarica 2013, Jahrg. 39, Nr. 2, S. 163-173. 


\begin{tabular}{|c|c|}
\hline $\begin{array}{l}\text { Cod. Hebr. } 5 \text { Bibel } \\
\text { (Hagiographen) })^{28}\end{array}$ & $\begin{array}{l}\text { f. 1r: [A. Agatha sancta] dixit si feras mihi promittis audito Christi nomine man- } \\
\text { suescunt (001308). A. Si ignem adhibeas rorem mihi salvificum de caelo angeli } \\
\text { ministrabunt (004897). A. Agatha laetissima et glorianter ibat ad carcerem et } \\
\text { quasi ad epulas invitata agonem suum domino precibus commendabat (001306). } \\
\text { R. Ipse me coronavit qui per apostolum Petrum in custodia me confortavit pro eo } \\
\text { quod jussa sum suspendi in equuleo propter fidem castitatis adju[va me domine } \\
\text { deus meus in tortura mamillarum mearum] (006990) } \\
\text { f. } 2 v \text { : [R. Qui me digna]tus est ab omni plaga curare et mamillas meas meo } \\
\text { pectori restituere lpsum invoco deum vivum (007479). V. Medicinam carnalem } \\
\text { (007479a). Ipsum. R. Beata Agathes ingressa carcerem expandit manus ad deum } \\
\text { et dixit [dixit domine qui me fecisti vincere tormenta carnificum jube me domine } \\
\text { ad tuam misericordiam pervenire](006160). } \\
\text { [Agathae] }\end{array}$ \\
\hline $\begin{array}{l}\text { Cod. Hebr. } 37 \text { Über } \\
\text { die Kräfte und } \\
\text { Wirkungen der } \\
\text { Nahrungs- und Heil- } \\
\text { mitte/29 }\end{array}$ & $\begin{array}{l}\text { f. 1r: [A. Hoc Joachim conturbatur confusus opprobrio quem pon]tifex templi pror- } \\
\text { sus notavit elogio (202210). A. Contribules et concives qui tunc illi aderant nequi- } \\
\text { vere contraire legi quam didicerant (200899). Ps. Fundavi. Riii. Inclyta stirps Jesse } \\
\text { virgam produxit amoenam de qua processit flos miro plenus [odore)] (601176) } \\
\text { [Annae] }\end{array}$ \\
\hline $\begin{array}{l}\text { Cod. Hebr. } 67 \text { The- } \\
\text { ologische Samme- } \\
\text { lhandschrift }\end{array}$ & $\begin{array}{l}\text { f. 1r: [A./R. Euge serve bone et fidelis quia in pauca] fuisti fidelis supra multa te } \\
\text { constitutam intra in gaudium domini dei tui }(002732,006677) * \text { In festo unius } \\
\text { virginis ad vs. A. Veni electa mea qui ponam in te thoronum (005322) } \\
\text { [Comm. unius Conf., Comm. Virginum] }\end{array}$ \\
\hline $\begin{array}{l}\text { Cod. Hebr. } 70 \mathrm{Hai} \\
\text { ben Joktan }\end{array}$ & $\begin{array}{l}\text { f. 1r: [A. In prole mater in partu virgo gaude et letare virgo mater] domini (003274). } \\
\text { A. Beata dei ge[netrix Maria virgo perpe]tua templum domini sacrarium spiritus } \\
\text { sancti [sola sine exemplo] placuisti domino Jesu Christo ora pro [populo interveni } \\
\text { pro clero] intercede pro devoto femineo sexu (001563). [A. O glo]riosa genetrix } \\
\text { virgo semper Ma[ria quae dominum omnium me]ruisti portare et regem archan- } \\
\text { gelor[um sola virgo lactare nostri quaesumus pia memorare et pro nobis Christum } \\
\text { deprecare ut tuis fulti patrociniis ad caelestia regna mereamur pervenire] (004029) } \\
\text { [Assumptio Mariae., Assumptio Mariae, 8] }\end{array}$ \\
\hline $\begin{array}{l}\text { Cod. Hebr. } 71 \text { Ma- } \\
\text { chzor nach deut- } \\
\text { schen Ritus }{ }^{32}\end{array}$ & $\begin{array}{l}\text { f. 1r: R. Nazarenus pater h[ujus vitae vir egregiae] mater autem de Bethlee[m } \\
\text { regali fulsit se]mine (601176). V. Dignum [enim erat talem] hoc oriri ordine } \\
\text { (601176a). [R. Quadam die] soli stanti ange[lus apparuit dicens] illi flere noli te } \\
\text { [deus exaudivit (602647). V. Sic] dolorem ejus gravem c[onfortans compescuit] } \\
\text { (602647a). R. Fir[ma fide* (600889). A. Ea quid]em tempestate praesul e[rat } \\
\text { Isachar qui Joachim infecundi refutabat munera] (201448) } \\
\text { [Annae] }\end{array}$ \\
\hline $\begin{array}{l}\text { Cod. } 2917 \\
\text { Sammelhandschrift } \\
\text { (Chronicon } \\
\text { Austriacum Incesti } \\
\text { Auctori) }\end{array}$ & $\begin{array}{l}\text { f. 1r: [V. Ex]pleta tunc oratione exauditur in agone domini promissione (600680a). } \\
\text { Post. R. Contristatus est praefectus regni scriba dum electus factus est catholicus } \\
\text { neque mundum neque deum timet morte necat eum homo diabolicus (600410). } \\
\text { V. Et livore justi tactus caritatis odit actus (600410a). Mundum. Gloria patri et Spi- } \\
\text { ritui Sancto. Laudes. A. Nobilis et formosa Dorothea generoso totius fraudis inscia } \\
\text { in Capadocie provincia imbuta [fide Christiana idola spernendo vana] (203260) } \\
\text { [Dorothea] }\end{array}$ \\
\hline
\end{tabular}

28 http://rosetta.nli.org.il/delivery/DeliveryManagerServlet?dps_pid=IE21223232

29 http://rosetta.nli.org.il/delivery/DeliveryManagerServlet?dps_pid=IE17332239

30 http://rosetta.nli.org.il/delivery/DeliveryManagerServlet?dps_pid=IE21223557

31 http://rosetta.nli.org.il/delivery/DeliveryManagerServlet?dps_pid=IE17332233

32 http://rosetta.nli.org.il/delivery/DeliveryManagerServlet?dps_pid=IE21346986 
Cod. 2918 Historische Sammelhandschrift f. 1r: Caeli* A. Valve matris integrales nulli viro subjugales hoc sunt portae aeternales (205991). Domini* W.* Specie tua et pulchritudine tua. Int. R. Arens virga fronduit radix Jesse floruit virgo deum genuit (604890). V. Ordo naturae non imperat huic geniturae (604890a).

[Mariae de Nive]

Tabelle 1 Fragmente des Ofner/ Pressburger Antiphonars, ÖNB (die Gruppe von Dr. F. Simader).

\section{Liebhard Egkenfelder}

Eine der interessantesten Persönlichkeiten der spätmittelalterlichen Pressburg war der hiesige Stadtnotar Liebhard Egkenfelder. Dieser Stadtschreiber, der von 1441 bis 1456 in Pressburg tätig war, war ein Laiengebildeter, eine humanistische Persönlichkeit seiner Zeit und ein fundierter Skriptor handschriftlicher Kodizes aus mehreren Wissenschaftsdisziplinen. Unter den von ihm abgeschriebenen Kodizes figurieren wertvolle Musikdenkmäler der kirchlichen und weltlichen Musikkultur. Über sein Leben und Wirken sind für mittelalterliche (oftmals anonyme) Verhältnisse, relativ viele Daten erhalten (Eintragungen in Stadtamtsbüchern, Testamenten, Skriptorenanmerkungen direkt in den von ihm abgeschriebenen Handschriften). Eine spezielle Informationsquelle über Liebhard Egkenfelders Leben und Werk ist sein Testament. ${ }^{33}$

Liebhard Egkenfelder (in den Quellen kommen verschiedene Formen seines Namens vor: Liebhart, Liphart Egkenueld, Ekkenfeld, Egkenvelt, Egkenuelter, Egkenvelter, Eckenfelder, Ekkenfelder), ${ }^{34}$ wurde in Niederbayern in dem Städtchen Eggenfelden geboren. ${ }^{35}$ Egkenfelders Geburtsdatum ist nicht bekannt. Da jedoch das Datum bekannt ist, wann er die Bakkalaureus-Würde an der Wiener Universität (3. Januar 1429) erwarb, kann laut Juraj Spiritza sein Geburtsdatum in den Jahren 1407-1411 angenommen werden.

Anhand einer Anmerkung auf f. 100v im Kodex/ Sammelhandschrift Cod. Ser. n. $3344^{36}$ der ÖNB wissen wir, dass er nach dem Abschluss des Bakkalaureusstudiums in Wien 1431 als Lehrer an der Stadtschule in Hainburg wirkte. ${ }^{37}$ Daneben war Egkenfelder in

33 Aus der Beschreibung der einzelnen Bücher der Bibliothek im Testament kann man teilweise z. B. die sogenannten Fakultätslehrbücher rekonstruieren, die Liebhard Egkenfelder während seines Studiums in Wien benutzte.

34 Wir verwenden die Namenform Liebhard Egkenfelder, die wir aus den Studien von Juraj Spiritza übernehmen. SPIRITZA, Juraj. Testament Liebharda Egkenfeldera, bratislavského mestského notára v rokoch 1441-1456. In Sbornik Slovenského národného múzea - História, 1967, roč. 61, S. 167-182; SPIRITZA, Juraj. Curriculum vitae bratislavského mestského notára a skriptora Liebharda Egkenfeldera. In SEDLÁK, Vincent (ed.). Zbornik príspevkom k slovenským dejinám. Bratislava: Slovenský historický ústav Matice slovenskej, 1998, S. $190-215$.

35 Diese Stadt ist in seinem Testament erwähnt, wo er seinem Cousin aus Eggenfelden 25 Gulden vermacht.

36 http://www.handschriftencensus.de/12228

37 Eine perfekte Analyse der Musikhandschrift Ser. n. 3344 aber auch des Lebens und der Tätigkeit Egkenfelders publizierte 2014 Marc Lewon. LEWON, Marc. Die Liedersammlung des Liebhard Eghenvelder: im Ganzen mehr als die Summe ihrer Teile. In RAUSCH, Alexander - TAMMEN, Bjorn R. (eds.). Musikalische Repertoires in Zentraleuropa (1420-1450). Wien, Köln, Weimar: Böhlau Verlag, 2014, S. 299-346. 
Hainburg auch als Lehrer in der Adelsfamilie Rukkendorf tätig. ${ }^{38}$ In Hainburg schrieb Liebhard Egkenfelder 1435 auch den Kodex mit dem Traktat von Nikolaus aus Dinkelsbühl (Sermones und andere theologische Texte) ab, der sich heute in der Bibliothek des Augustiner-Chorherrenstift in Klosterneuburg (Cod. 486) befindet. ${ }^{39}$ In der Handschrift findet sich an mehreren Stellen die Anmerkungen, dass der gegebene Teil von Liebhard selbst und zwar in Hainburg und während seines Aufenthaltes im Hause Rukkendorfer geschrieben wurde.

Liebhard Egkenfelder wirkte in Hainburg wahrscheinlich 5 Jahre. Während seines Aufenthaltes in dieser Stadt heiratete er eine gewisse Anna Mautner aus Pressburg, wobei ihn seit 1439 auch der Besitz eines Weinbergs mit Pressburg verband. Zwischen seinem Wirken in Hainburg und später in Pressburg arbeitete er als Hilfs- und danach als Stadtschreiber in Sopron. Im März 1441 holte der Pressburger Stadtrat Liebhard Egkenfelder in seine Dienste. Davon zeugt eine Ausgabe für die Zustellung eines Schreibens von Pressburg nach Sopron, die im Kammerbuch aus dem gegebenen Jahr verzeichnet ist. Als Stadtschreiber wirkte Egkenfelder in Pressburg vom 3. Juli 1441 bis zum 5. April 1456, also fast 15 Jahre. ${ }^{40}$ Liebhard Egkenfelder war ein geachteter Stadtbürger Pressburgs. Als Schreiber half er in der Bruderschaft Corporis Christi aus, deren Mitglied er seit 1445 war. Seit 1448 war er Verwalter (Hofmeister) der Zisterzienserkurie bei der Kapelle St. Katharina. Liebhard Egkenfelders Schreibertätigkeit bestand in der schriftlichen Aufzeichnung der laufenden Agenda der Stadt, der Gerichtsagenda, der Führung der Stadtbücher und in der Anfertigung auch anderer Schriftstücke, wofür er ein Gehalt erhielt. Er vertrat Pressburg in Gerichtsprozessen und war Zeuge vieler testamentarischer Entscheidungen.

Ein höchst interessanter Beleg über die außergewöhnliche Persönlichkeit Liebhard Egkenfelders ist das erwähnte Testament (Protokoll der Testamente Pressburgs aus den Jahren 1427-1529, 4nl, 90-93 ${ }^{\mathrm{r}}$. PPT 1., Nr. 183, 241-248). ${ }^{41}$ Das lange und interessant konzipierte Dokument zeugt von einem umfassend gebildeten Mann, der außer seinem Beruf eines Schreibers der Stadtkanzlei auch eine komplexe Renaissance-Persönlichkeit seiner Zeit war. Egkenfelder fertigte es 1455 nach dem Tod seiner Frau an. Das Original des Testaments ging verloren. Seinen ganzen Wortlaut enthält jedoch die Abschrift vom 13. Dezember 1457. Das Dokument ist ungewöhnlich umfangreich und ist in zwei Teile gegliedert. Es besteht aus dem Vermögensverzeichnis (Bücher, Kleidung, Stoffe, Waffen, Werkzeug, Schmuck, Immobilien, Mobiliar) und dem eigentlichen Testament.

38 Wahrscheinlich auf einen Hinweis von Georg/Jörg Rukkendorf schrieb Liebhard Egkenfelder in Hainburg die Handschrift der ÖNB Ser. $n .3344$ ab. Dieser Kodex mit seinem bunten Inhalt ist für den Musikhistoriker von Bedeutung, vor allem hinsichtlich der Erforschung des Repertoires des deutschen Minnesangs. Den musikalischen Teil der Handschrift untersucht Marc Lewon eingehend in seiner Studie von 2014, und ihren musikalischen Inhalt bringen wir im weiteren Teil der Studie näher.

39 http://manuscripta.at/hs_detail.php?ID=656

40 Der Schreiberhand Liebhard Egkenfelders und seiner Helfer widmet sich eine Studie von Darina Lehotská aus dem Jahr 1958. LEHOTSKÂ, Darina. Vývoj bratislavskej mestskej kancelárie do roku 1523. Historické štúdie 1958, Jahrg. 4, S. 222-274.

41 MAJOROSSY, Judit - SZENDE, Katalin (eds.). Das Pressburger Protocollum Testamentorum 1410 (1427)-1529, Teil 1: 1410-1487. Wien: Böhlau Verlag, 2010. 
Besondere Aufmerksamkeit lenken hinsichtlich seiner Skriptoren-, konkreter aus musikologischer Sicht - Notatorentätigkeit, vor allem Egkenfelders Erwähnungen seiner Bücher auf sich. In seinem Testament führt Liebhard Egkenfelder 37 Bücher an, die ihm gehörten. Hinzu fügt er noch die Erwähnung weiterer 4 Bücher, die er der Pfarre St. Martin geliehen hatte. Und weitere vier Kodizes, die er im Zusammenhang mit seinen künftigen Besitzern erwähnt. Die Gesamtzahl der in Liebhard Egkenfelders Testament erwähnten Bücher beträgt somit die Nummer 45. Von dieser Zahl hatte er einen Kodex käuflich erworben, 3 bekommen und 4 ausdrücklich nicht selbst geschrieben.

In dem Testament sind Bücher religiösen und rechtlichen Charakters, philosophische, historische, medizinische Werke und schöngeistige Literatur, sogar ein deutsches Schachhandbuch (schön gemalt) beschrieben. Besonders interessant sind auch die detaillierten Beschreibungen der einzelnen Bücher.

Sie betreffen zum Beispiel den Einband - „in rotes Wildleder gebunden“ (ist in ain rot sëmische vel gebunden), Ausschmückungen - „schön illuminierte Handschrift mit der Abbildung der Jungfrau Maria am Anfang (schon illuminiret, am anfang Marie pildung), Spezifikationen des Inhalts (Offizium - Geschichte zum Fest des Besuchs der Jungfrau Maria) und das Material der Handschrift (in fine histori von Unser Frawn Visitationis papirein), die Herkunft (daz hat mir geschannkt her Peter Zistler in gegenbürt herrn Mert, pharrern S. Martini), mit der Angabe, dass der Kodex notiert ist (notel geschrif) oder mit der Information, dass er die Handschrift mit eigener Hand geschrieben oder nicht geschrieben (meiner hanntgeschrifft) hat.

Laut Testament hat Liebhard Egkenfelder nachweislich 16 Handschriften geschrieben. Bei 21 Kodizes führt er die Herkunft nicht an. ${ }^{42}$

Aus dem Testament selbst geht hervor, dass Egkenfelder eine interessante und zahlreiche Sammlung liturgischer Bücher besaß. Das Evangeliar kaufte er von dem Pressburger Kanoniker Christian Leyth (Item ain puech in pergamen und text geschriben in ainer weissen ligatur, darinn man zu kor das ewangelium list, und habs kaufft von herrn Cristan Korherrn, der im spital starb, Kodex Nr. 11), ein Lektionar mit nicht genannter Herkunft (Item ain chlains pühel in pergamen gar gueter geschrifft, und ist ein leccionarius durch das ganncz jar mit schon legenden, in pretel gepunden und mit aim plaben vel uberczogen und beslagen, Kodex Nr. 25), 2 Psalter, von denen einer an seinem Anfang mit der Darstellung der Jungfrau Maria illuminiert war (Item zway selterel, der ain gar chlain mit aim weissen semisch uberczogen, in pergamen, text geschriben, schon illuminiret, am anfang Marie pildung, der ander auch in pergamen, text geschrifft, und ist ain taill grosser mit ainem roten fel uberczogen und beslagen, Kodizes Nr. 27 und 28), das Diurnal mit Gebeten zum Fest des Besuchs der Jungfrau Maria, das Egkenfelder 1454 als testamentarischer Zeuge von dem Pressburger Kaplan Peter Zistler erhalten hatte (Item ain swarcz diurnal von herrnn Peter Zistler, in pergamen und text geschriben, in fine histori von Unser Frawn Visitationis papirein, Kodex Nr. 32).

Besonders interessant ist die Erwähnung des Besitzes eines Gebetbuchs mit Noten, das ebenfalls ein Geschenk von Kaplan Zistler an ihn war (Item ain pettpüchel in pergamen als regel pleter, notel geschrift, darin XV gradus und ander vil oraciones, mit aim plaben vel

42 Mindestens 2 weitere schrieb er jedoch auch während seines Wirkens in Österreich - eine davon, die Wiener Sammelhandschrift Cod. Ser.n. 3344 war bestimmt kein Bestandteil des Testaments. 
uberczogen, daz hat mir geschannkt her Peter Zistler in gegenbürt herrn Mert, pharrern S. Martini, Kodex Nr. 32). Laut Testament besaß Egkenfelder noch einen weiteren notierten Kodex, und zwar einen auf Papier geschriebenen Kodex - "Specialnik", teils Kantional und teils Graduale, den Egkenfelder selbst angefertigt hatte (Item ain special genotirt in regal papir, und ist ain cantual ains gradual, meiner hanntgeschrifft, Kodex Nr. 36). Diese Aufzeichnung bestätigt Egkenfelders Fähigkeit, auch notierte Teile der Handschriften und somit auch notierte Kodizes zu schreiben (abzuschreiben).
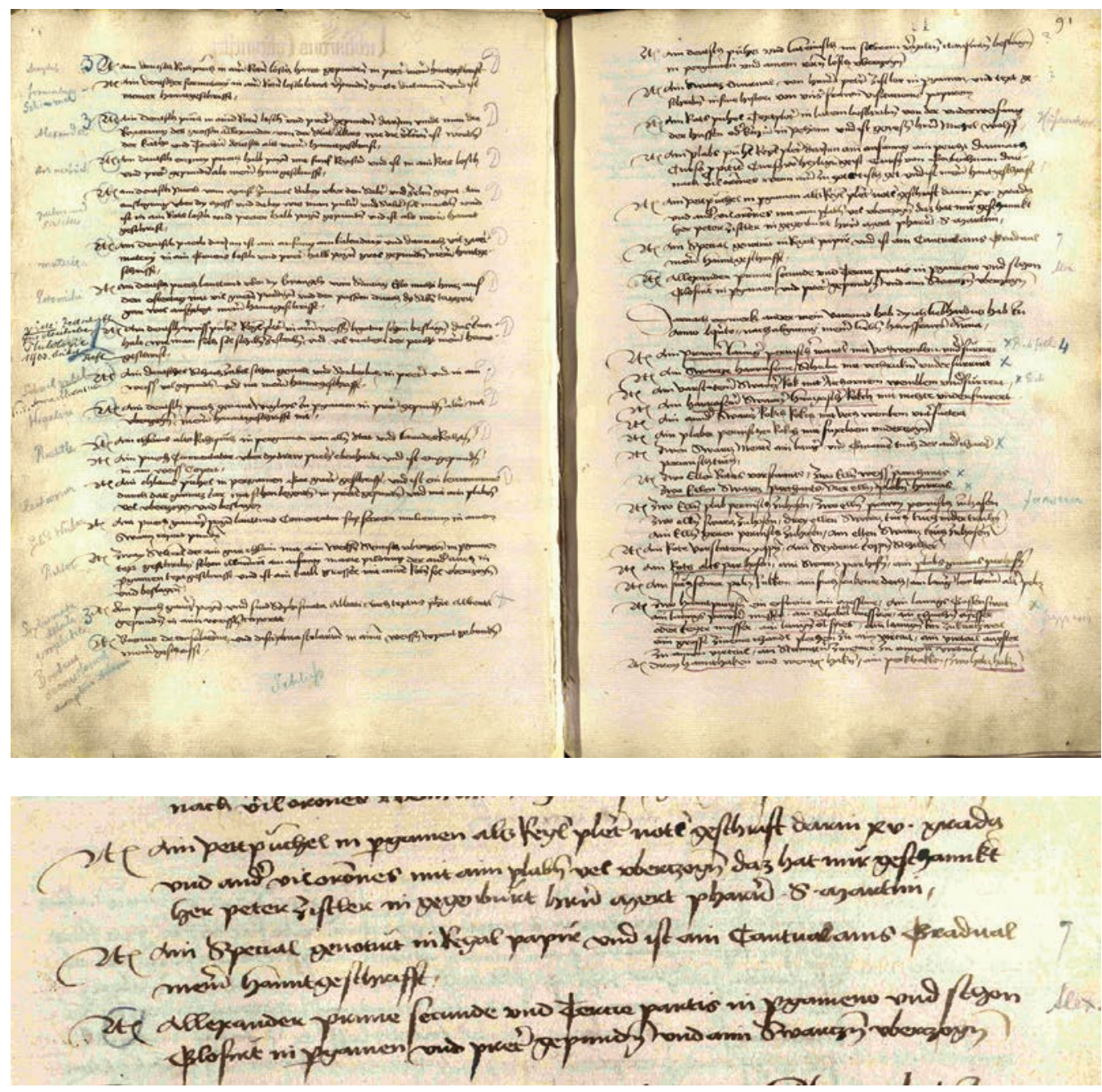

Abb. 4 Das Pressburger Protocollum Testamentorum 1410 (1427) - 1529 (Archiv der Stadt Pressburg), f. 90r-93v:43

43 http://www.crarc.findbuch.net/php/main.php?ar_id=3738; https://www.crarc.findbuch.net/php/view. php?link=414d422d412f585849562e31x156\#\&rotation=6.283185307179586\&path=d3c7c76bd0d5dcc76b- 
Während seiner Zeit in Pressburg kann bei Egkenfelder neben seinem Interesse für liturgische Handschriften und der Fähigkeit, liturgische Musik abzuschreiben, auch das Interesse für das weltliche Lied des deutschen Minnesangs nachgewiesen werden. Davon zeugt eine Bemerkung im Testament bei dem Kodex Nr. 15: Item ain deutsch puch in ainem roten losch und preter gepunden, darinn vindt man die regierung des grossen Allexander, von der stat Akers, wie die verloren ist worden, der Katho und Teichner deutsch, als meiner hanntgeschrift (Kodex Nr. 15), die über den Inhalt der Handschrift informiert, in der sich unter anderem auch ein nicht näher bekannter Text des Minnesängers Heinrich Teichner (um 1310 - vor 1378) befunden haben soll.

Die bewundernswerte Bibliothek Liebhard Egkenfelders zerfiel nach dem Tod ihres Besitzers, da er sie vor seinem Tode im Rahmen seines testamentarischen Vermächtnisses verschenkt hatte. Seine Bücher widmete Liebhard Egkenfelder nicht nur den genannten Personen aus Pressburg und Umgebung (beschenkt wurden z. B. Johannes Schuß, Wennczlaus Pernnhartel, Jörg und Wolfgang Ranes, Michel von Newsidel, Christoph, Leonhard Planckh, Kungsfelders Sohn, Doktor Wolff), sondern auch Institutionen dem Paulinerkloster in Marianka (Mariental), der Kirche St. Martin und der Schule für die Bedürfnisse armer Studenten.

Bis heute konnten nur vier erhaltene Handschriften identifiziert werden, die Egkenfelder schrieb bzw. besaß.

Das sind die zwei österreichischen Kodizes - Sammelhandschrift Cod. Ser. n. 3344 aus der ÖNB und das Traktat von Nikolaus von Dinkelsbühl Cod. 486 aus der Bibliothek der Augustiner-Chorherrenstift in Klosterneuburg, ${ }^{44}$ ferner Postillen und Predigten (Ars moriendi) Mus. 1 aus der Ungarischen Franziskanerbibliothek in Budapest ${ }^{45}$ und der Kodex Br 8879-8880 aus der Königlichen Bibliothek in Brüssel (Bibliothèque Royal Brüssel) ${ }_{.}^{46}$ Die letzte der genannten Handschriften ist auch hinsichtlich der Kulturgeschichte Ungarns und Pressburgs interessant, da sie außer der geistlichen lateinischen und weltlichen deutschen Poesie zwei Seiten mit Egkenfelders persönlichen Bemerkungen mit verschiedenen chronikarischen Reaktionen auf historische und politische Ereignisse aus den Jahren 1451 - 1453 enthält. ${ }^{47}$

f76ed26be0e0ece16beee3ec6be0e0ede4ed6bed37e0c76bf76ed26be0e0ece16beee3ec6be0e0ede4ed6bed37e06be1eae0c739f13f

44 BORECZKY, Anna. Details des Bilderkatechismus der Concordantiae caritatis im Klosterneuburger Kodex von Liebhardt Egkenfelder. In Galéria, 2004-2005, Bratislava: Slovenská národná galéria, S. 161-166.

45 Ibidem.

46 Der Kodex enthält auch eine deutsche Ars moriendi und die Abhandlung von Heinrich von Langstein. Laut Lewon handelt es sich um den Kodex Nr.18. Wir sind der Ansicht, dass es sich eher um die Handschrift Nr. 8 oder 10 handelt. LEWON, 2014, op. cit., S. 299-346. SPIRITZA, 1967, op. cit., S. 179-181.

47 Die Bemerkungen betreffen zum Beispiel die Beschreibung der Kämpfe von Johann Hunyady (13871456) und Johann Giskra (1400-1469). Spezielle Aufmerksamkeit wird dabei den Ereignissen der Adventszeit des Jahres 1452 gewidmet, als sich in Pressburg das ungarische Heer versammelte. Vom Anfang des Jahres 1453 wird der Einzug des Königs Ladislaus Postumus (1440-1457) in Wien erwähnt, wo während der Zeremonie Johann Hunyady der Grafentitel verliehen wurde. Im selben Jahr wird die feierliche Rückkehr des Königs nach Pressburg am Freitag vor dem Fest der Bekehrung des hl. Paulus (25. Januar 1453) und seine Rückkehr nach Wien am Mittwoch nach dem Fest der hl. Dorothea (6. Februar 1453) erwähnt. Datierung und Beschreibung des Ereignisses befinden sich auf Folio 24 , unmittelbar vor dem dreistrophigen deutschen Lied Carmen 
Egkenfelders Skriptorenaktivitäten sind also belegbar im Bereich der weltlichen und kirchlichen Musikkultur. Egkenfelder schrieb und notierte nachweislich selbst zwei Musikhandschriften (wie bereits erwähnt: eine führt er selbst im Testament als Kodex „Specialnik" an, diese Handschrift ist leider nicht erhalten). In der österreichischen Nationalbibliothek in Wien befindet sich jedoch sein Kodex Sammelhandschrift Cod. Ser. n. 3344,48 der während Egkenfelders Aufenthalts in Hainburg entstand. ${ }^{49}$

In diesem sog. Hausbuch befinden sich Aufzeichnungen sogar von 12 Neidhart-Liedern, daher ist es in den deutschsprachigen Ländern auch als Wiener Neidharthhandschrift „w" " bekannt. $^{50}$

Der Liedteil des Kodex beginnt direkt auf der letzten Seite der Chronik, was die Tatsache bestätigt, dass die notierten Teile nie ein eigenes Buch gebildet haben, sondern ein integraler Bestandteil der kompletten Handschrift (Sammelhandschrift) waren.

Der notierte Teil, die sog. „Eghenvelder-Liedersammlung“, nimmt in der deutschen sprach- und musikwissenschaftlichen Literatur einen besonders wichtigen Platz ein. Er ist eine der wichtigen Vergleichsquellen verschiedener Genres der deutschen mittelalterlichen Lyrik. In der Egkenfelder Sammlung befinden sich insgesamt 31 Texte deutscher Lieder, wobei zu jeder ersten Strophe auch Notenlinien für die Eintragung der Melodie vorbereitet sind. Die Melodie ist bei 20 Liedern eingetragen (davon bei zweien nur fragmentarisch) und bei 10 Liedern blieben die vorbereiteten Notenlinien leer. Die meisten leeren Linien sind in den letzten Teilen der Sammlung. Nach Lewon beendete Egkenfelder die Sammlung nicht und verließ Hainburg noch bevor sie fertiggestellt war. Eine weitere Möglichkeit konnte auch die Tatsache sein, dass er zu einigen ausgewählten Liedern kein Musterexemplar besaß. Verwendet wurden 3 Notationstypen: der Choral-, der Mensural- und der Übergangstyp mit dem Choral-Mensuralsystem. Von dem „Neidhartschen" Hauptkorpus befinden sich in dieser Handschrift 12 Neidhartsche Lieder,

\footnotetext{
p[ro] ingressu Regis Ladi[slai] wienne compositu[m], das auf die erwähnten historischen Ereignisse an der Jahreswende 1452/1453 reagiert. Die Aufzeichnung des Liedes ist leider ohne Melodie. Nach Franz Maschek (diese Information betrachten wir jedoch als unzureichend mit Quellen belegt) wurde das Lied bei dem festlichen Einzug von König Ladislav Pohrobok in Pressburg und Wien in deutscher Sprache gesungen. MASCHEK, Franz. Die Handschriftensammlung des Stadtschreibers Liebhard Eghenvelder. Unsere Heimat, Monatsblatt des Vereins für Landeskunde von Niederösterreich und Wien, 1953, S. 93-96.

48 MAZAL, Otto - UNTERKIRCHER, Franz. Katalog der abendländischen Handschriften der Österreichischen Nationalbibliothek: Series nova (Neuerwerbungen): Teil 3: Cod. Ser. n. 3201-4000. Wien: Prachner, 1967, s. 67-84. http://www.handschriftencensus.de/12228; http://www.cantusplanus.at/de-at/projektphp/signatur2.php?Signatur=sn03344; http://www.manuscripta.at/hs_detail.php?ID=7017

49 Die Sammelhandschrift besteht aus mehreren Teilen: auf den ersten acht Folien sind Texte über Wappen, gefolgt von Leopold Stainreuters Österreichischer Chronik von den 95 Herrschaften, danach folgen notierte Aufzeichnungen von Liedern bayrischer Minnesänger und am Schluss des Kodex befinden sich noch weitere Texte unterschiedlichen Inhalts. Schon in der zweiten Hälfte des 15. Jahrhunderts war die Handschrift im Besitz von Jörg Schrat, dem Pfarrer der Peterskirche in Wien, dessen Name in der Handschrift mehrfach hinzugefügt wurde (einmal auch mit der Jahresangabe 1457). 1883 wurde der Kodex von einem gewissen Josef Rettl aus Steyregg für die Hofkirche in Wien gekauft. Aus sprachwissenschaftlicher und musikalischer Sicht besonders wertvoll sind vor allem die Abschriften deutscher Lyrik, darunter Gedichte und Lieder von Peter von Arberg, Reinmar von Brennenberg, Neidhart von Reuenthal und anderer.
}

50 LOMNITZER, Helmut. Liebhard Eghenvelders Liederbuch. Zeitschrift für die deutsche Philologie, 1973, Jahrg. 90, S. 190-216. 
von denen 9 notiert sind. Ferner befinden sich in der Handschrift auch das Lied eines Mönchs aus Salzburg (Maria ward ein pot gesandt), zwei Lieder von Reinmar von Brennenberg und das anonyme Lied Gegrusset seistu, Maria, du uil raine maid. Die Mehrheit der Lieder ist ohne Angabe des Autors. Fast ein Drittel beinhalten jedoch spezifische Angaben und Anmerkungen, die den Liedautor identifizieren helfen. Das sind z.B. die Anmerkungen Brenberger wei $\beta\left(112^{\mathrm{r}}\right)$, tagweis $\left(102^{\mathrm{v}}\right)$, der munich $\left(113^{\mathrm{v}}\right)$, Neidhart $\left(109^{\mathrm{v}}\right)$. Die Zusammenstellung der gesamten Liedersammlung ist gut durchgearbeitet und durchdacht, sie hat aber nicht den repräsentativen Charakter eines pompösen Buchkodex, sondern eher den einer praktischen Handschrift für den persönlichen Bedarf des Benutzers oder Auftraggebers.

Bei einer Gesamtbetrachtung dokumentiert die Liedersammlung von Liebhard Egkenfelder einen gemischten Charakter des Repertoires und präsentiert spezifische Qualitäten ihres Schöpfers. Sie ist aus verschiedenen Quellen der deutschen mittelalterlichen Lyrik zusammengestellt, wobei sie ein lebendiges, beliebtes und aktuelles Repertoire ihrer Zeit aus dem Milieu Wiens und seiner Umgebung widerspiegelt. Die Sammlung präsentiert die zeitgemäßen Kenntnisse des Autors und die praktischen Beweggründe des Schreibers. Die Liederauswahl präsentiert das Zeitrepertoire des studentischen Umfelds der Wiener Universität, das Egkenfelder während seines Universitätsstudiums gewiss kennengelernt hat. ${ }^{51}$

Liebhardt Egkenfelder zwar zweifellos ein äußerst gebildeter Mensch, der neben den hervorragenden Schreibfähigkeiten auch die Notenschrift beherrschte. Diese Behauptung bezeugen direkt die testamentarische Eintragung über den notierten Kodex - den sog. Specialnik und die Handschrift Cod. Ser. n. 3344 aus der ÖNB. Wir nehmen an, dass Egkenfelder die Choralschrift und die Mensuralnotenschrift beherrschte. Die Wiener Sammelhandschrift dokumentiert mehrere Notationen: die Metzer-gotische Choralnotation, die Mensuralnotation und die gemischte Mensural-Choralnotation, die Egkenfelder wahrscheinlich von Musterhandschriften abschrieb. Einen besonders verwandten Typ der abgeschriebenen Handschrift, geschaffen ebenfalls aus mehreren Mustern, stellt zum Beispiel die Tyrnauer Handschrift Clmae 243 (Ungarische Nationalbibliothek in Budapest) dar, die ähnlich mehrere Genre- und Notationskreise der lateinischen, deutschen und böhmischen liturgischen Musik nicht nur in lateinischer, sondern auch in den Nationalsprachen umfasst. ${ }^{52}$

51 LEWON, 2014, op. cit., S. 299-346.

52 Die vorliegende Studie wurde im Rahmen der Studienbeihilfen VEGA 2/0034/17 „Obraz zbožnosti v stredovekej hudobnej kultúre na Slovensku“ [Die Frömmigkeit in der mittelalterlichen Musikkultur in der Slowakei] (2017-2020) und Nr. APVV-19-0043 „CANTUS PLANUS in der Slowakei - lokale Elemente und transregionale Beziehungen“ (2020-2024) bearbeitet. Die Übersetzung in die deutsche Sprache wurde von Margita Kurdel realisiert. 


\section{Bibliography}

BORECZKY, Anna. Details des Bilderkatechismus der Concordantiae caritatis im Klosterneuburger Kodex von Liebhardt Egkenfelder. In Galéria, 2004-2005, Bratislava: Slovenská národná galéria, S. 161-166.

BURAN, Dušan. „Böhmisch - slowakisch - österreichisch“? Die Malerei in Pressburg (Bratislava). In Böhmische Buchmalerei des 14. und 15. Jahrhunderts. Purkersdorf: Verlag Brüder Hollinek, 2012, S. 45-55.

BURAN, Dušan. Fragmente illuminierter gotischer Handschriften aus zwei slowakischen Archiven. In Biblos. Beiträge zu Buch, Bibliothek und Schrift Europa, Die Nationalbibliotheken der neuen EU-Länder. Wien: Phoibos Verlag, 2004, S. 67-79.

BURAN, Dušan. Neznámy budínsky (?) iluminátor a maliar z okruhu Ulricha Schreiera. In RUSINA, Ivan et al. (eds.). Renesancia: umenie medzi neskorou gotikou a barokom: Dejiny slovenského výtvarného umenia. Bratislava: Slovenská národná galéria - Slovart, 2009, S. 873-874.

DOBSZAY, László. A »Budai antiphonále« megtalált töredékei. Zenetudományi dolgozatok. Budapest: MTA Zenetudományi Intézet, 1978, S. 35-39.

HAIDINGER, Alois. Bemerkungen zur Entstehung des Großen Antiphonars Klosterneuburg, Stiftsbibliothek, Cod. 65-68. In Böhmische Buchmalerei des 14. und 15. Jahrhunderts. Purkersdorf: Verlag Brüder Hollinek, 2012, S. 73-80.

HAIDINGER, Alois. Katalog der Handschriften des Augustiner Chorherrenstiftes Klosterneuburg. Teil 1: Cod. 1-100. Wien: Österreichische Akademie der Wissenschaften, 1983.

HLAVAČKOVÁ, Miriam. Juraj zo Schönbergu. Bratislavský prepošt v službách cisára a krála. Bratislava: VEDA - Historický ústav SAV, 2015.

HLAVAČKOVÁ, Miriam. Kapitula pri Dóme sv. Martina. Intelektuálne centrum Bratislavy v 15. storoč́. Bratislava: Pro Historia, 2008.

HLAVAČKOVÁ, Miriam. Oltárne benefíciá v bratislavskom Dóme sv. Martina v 15. storočí. In Galéria, 2001, Bratislava: Slovenská národná galéria, 2001, S. 85-99.

HLAVAČKOVÁ, Miriam. Vzdelanostná úroveň členov Bratislavskej kapituly v 15. storočí. Historický časopis 2001, 49, Nr. 3, S. 433-451.

KLUGSEDER, Robert - RAUSCH, Alexander - ČIZMIĆ, Ana - CHARVAT, Vera Maria -VERHAAR, Oscar -VESELOVSKÁ, Eva - ZÜHLKE, Hanna. Katalog der mittelalterlichen Musikhandschriften der Österreichischen Nationalbibliothek Wien. In Codices Manuscripti Ẽ Impressi. Purkersdorf: Verlag Brüder Hollinek, 2014, Supplementum 10.

KLUGSEDER, Robert. Medieval musical manuscripts and fragments in the Austrian National Library (ÖNB), Vienna. In Cantus Planus. Papers read at the 16th Meeting Vienna, 2011. Wien: Verlag Brüder Hollinek, 2012, S. 192-201.

LEHOTSKÁ, Darina. Vývoj bratislavskej mestskej kancelárie do roku 1523. Historické štúdie 1958, Jahrg. 4, S. 222-274.

LEWON, Marc. Die Liedersammlung des Liebhard Eghenvelder: im Ganzen mehr als die Summe ihrer Teile. In RAUSCH, Alexander - TAMMEN, Bjorn R. (eds.). Musikalische Repertoires in Zentraleuropa (1420-1450). Wien, Köln, Weimar: Böhlau Verlag, 2014, S. 299-346.

LOMNITZER, Helmut. Liebhard Eghenvelders Liederbuch. Zeitschrift für doe deutsche Philologie, 1973, Jahrg. 90, S. 190-216.

MAJOROSSY, Judit - SZENDE, Katalin (eds.). Das Pressburger Protocollum Testamentorum 1410 (1427)-1529. Teil 1: 1410-1487. Wien: Böhlau Verlag, 2010.

MASCHEK, Franz. Die Handschriftensammlung des Stadtschreibers Liebhard Eghenvelder. Unsere Heimat, Monatsblatt des Vereins für Landeskunde von Niederösterreich und Wien, 1953, S. 93-96. 
MAZAL, Otto - UNTERKIRCHER, Franz. Katalog der abendländischen Handschriften der Österreichischen Nationalbibliothek: Series nova (Neuerwerbungen): Teil 3: Cod. Ser. n. 3201-4000. Wien: Prachner, 1967.

MIKÓ, Árpád. Fáncsi Imre armálisa (1511) és két Budán illuminált, kottás díszkódex. Ars hungarica 2013, Jahrg. 39, Nr. 2, S. 163-173.

RISCHPLER, Susanne. Der Illuminator Michael. Codices Manuscripti. Supplementum 1, 2009. Purkersdorf: Verlag Brüder Hollinek, 2009.

SOPKO, Július. Stredoveké latinské kódexy slovenskej proveniencie v Mad’arsku a Rumunsku. Martin: Matica slovenská, 1982.

SOPKO, Július. Stredoveké latinské kódexy v slovenských knižniciach. Martin: Matica slovenská, 1981.

SPIRITZA, Juraj. Curriculum vitae bratislavského mestského notára a skriptora Liebharda Egkenfeldera. In SEDLÁK, Vincent (ed.). Zbornik príspevkom k slovenským dejinám. Bratislava: Slovenský historický ústav Matice slovenskej, 1998, S. 190-215.

SPIRITZA, Juraj. Testament Liebharda Egkenfeldera, bratislavského mestského notára v rokoch 1441-1456. In Sbornik Slovenského národného múzea - História, 1967, roč. 61, S. 167-182.

SZENDREI, Janka. A magyar középkor hangjegyes forrásai. Budapest: MTA Zenetudományi Intézet, 1981.

SZENDREI, Janka. Die Geschichte der Graner Notation. Studia Musicologica Academiae Scientiarum Hungaricae 1988, 30, S. 5-234.

ŠEDIVÝ, Juraj. Beiträge zur mittelalterlichen deutschprachigen Schriftkultur in der Slowakei I. Bratislava: Univerzita Komenského, 2019.

ŠEDIVÝ, Juraj. Mittelalterliche Schriftkultur im Pressburger Kollegiatkapitel. Bratislava: Chronos, 2007. THEISEN, Maria - JENNI, Ulricke (eds.). Mitteleuropäische Schulen IV (ca. 1380-1400). Hofwerkstätten König Wenzels IV. und deren Umkreis. Wien: Verlag der Österreichischen Akademie der Wissenschaften, 2014.

VESELOVSKÁ, Eva - ADAMKO, Rastislav - BEDNÁRIKOVÁ, Janka. Stredoveké pramene cirkevnej hudby na Slovensku. Bratislava: Slovenská muzikologická spoločnost’ - Ústav hudobnej vedy SAV, 2017.

VESELOVSKÁ, Eva. Bohemian Notation in Slovakia in the Middle Ages. Hudební věda, 2012, Jahrg. 49, Nr. 4, S. 337-376.

VESELOVSKÁ, Eva. Catalogus fragmentorum cum notis musicis medii aevi e civitate Tyrnaviensi. Catalogus fragmentorum cum notis musicis medii aevi in Slovacia. Tomus IV. Bratislava: Ústav hudobnej vedy SAV, 2015.

VESELOVSKÁ, Eva. Der böhmische Einfluß auf mitteleuropäische Musikhandschriften des 14. und 15. Jahrhunderts unter Berücksichtigung illuminierter Codices. In Böhmische Buchmalerei des 14. und 15. Jahrhunderts. Purkersdorf: Verlag Brüder Hollinek, 2012, s. 95-115.

VESELOVSKÁ, Eva. Fragmente des Budaer Antiphonars im St. Adalbert-Verein Trnava und im Archiv des Slowakischen Nationalmuseums. Studia Musicologica, 2015, Jahrg. 56, Nr. 2-3, S. 233-246.

VESELOVSKÁ, Eva. Métsko-gotická notácia na Slovensku v období stredoveku. Musicologica Slovaca, 2018, Jahrg. 9 (35), Nr. 2, S. 165-199.

VESELOVSKÁ, Eva. Mittelalterliche liturgische Kodizes mit Notation in den Archivbeständen von Bratislava. Ed. Musaeum Musicum. Bratislava: Slovenské národné muzeum - Hudobné muzeum, 2002. 